\title{
A Proposal for the Study of Laminar Relativistic Electron Beam Generation by a Foilless Diode
}

\author{
Michael E. Jones \\ Lester E. Thode
}
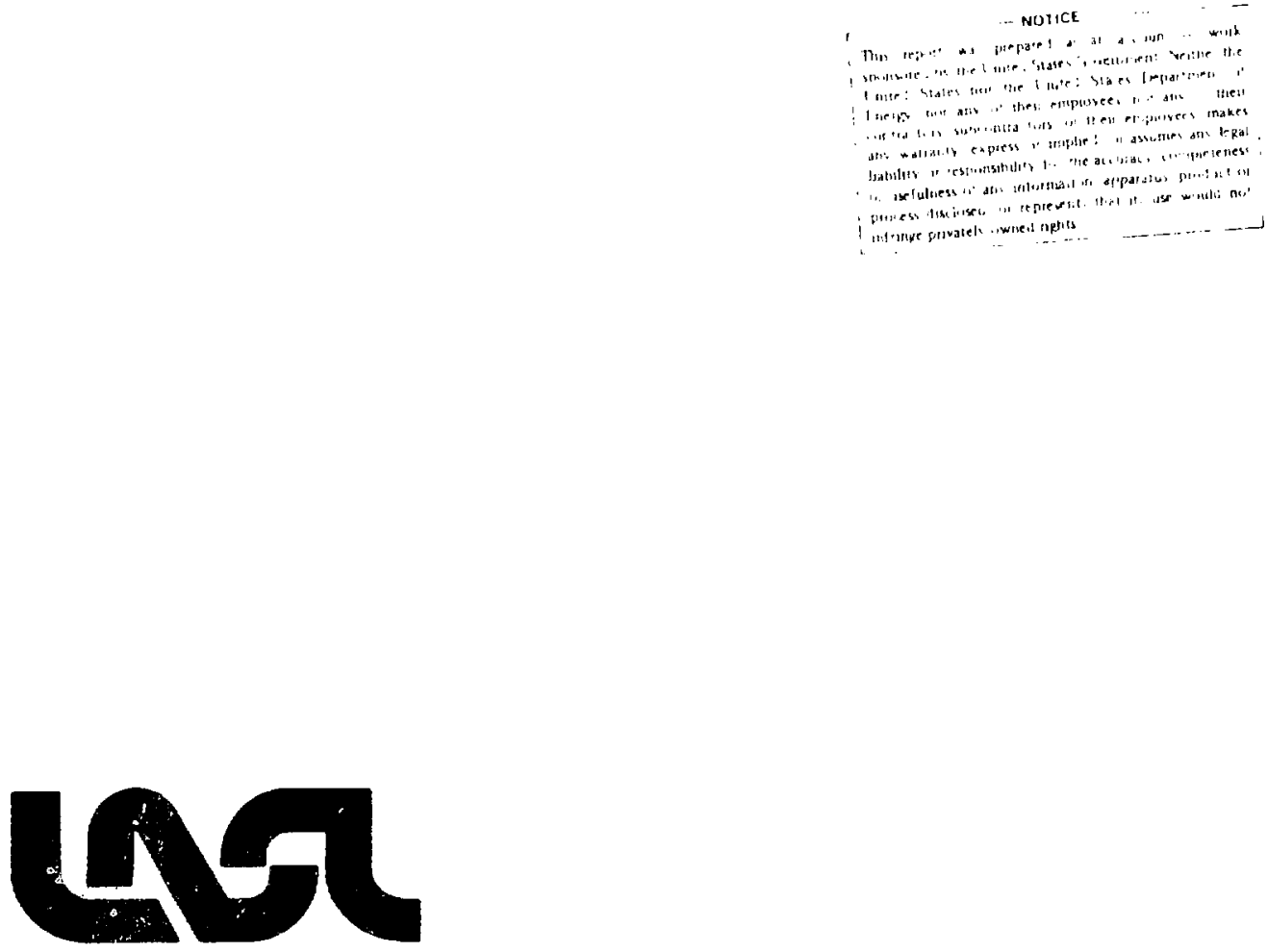


\section{A PROPOSAL FOR THE STUDY OF LAMINAR RELATIVISTIC ELECTRON BEAM GENERATION BY A FOILLESS DIODE}

by

Michael E. Jones and Lester E. Thode

\section{ABSTRACT}

The continuation of an analytical and numerical study of intense relativistic electron beam generation by foilless diodes is proposed. The investigation is aimed at optimizing the diode design to produce a laminar flow.

\section{INTRODUCTION}

A theoretical investigation into the generation of intense relativistic electron beans by foilless diodes is currently being conducted at Los Alamos Scientific Laboratory (LASL) under contract to the Air Force Office of Scientific Research (AFOSR). 1 The goal of the study is to determine the feasibility of using a foilless diode to produce a 5 to $50 \mathrm{MeV}$ electron beam for several applications, including high-density plasma heating, submillimeter microwave generation, and collective ion acceleration.

In conjunction with a 5 to $50 \mathrm{MeV}$ particle energy, these applications ultimately require a beam energy density of $10^{19}$ to $10^{22} \mathrm{eV} / \mathrm{cm}^{3}$. This implies a current density of 50 to $500 \mathrm{kA} / \mathrm{cm}^{2}$, but not necessarily a large net current. Furthermore, the local beam distribution function must be sufficiently uniform in energy and well collimated, since the applications of interest depend on the development of large-amplitude, coherent waves. In this context, a measure of the beam quality is the spread in electron velocity components along a beam stream line,

$$
\Delta v_{11} / c \cong \theta^{2} / 2+\delta \gamma / \gamma^{3},
$$


where $\theta$ and $\delta \gamma$ are the characteristic angular and energy spread of the local beam distribution function, and $\gamma$ is the Lorentz factor. The magnitude of $\Delta v_{\text {II }}$ which can be tolerated depends upon the wave spectrum associated with the particular application. For example, high-density plasma heating requires that $\theta \leqq 30 \mathrm{mrad}$ or equivalently

$$
\Delta v_{1} / c \leq 4 \times 10^{-4} \lesssim 4 \gamma^{-1}\left(n_{b} / 2 n_{p}\right)^{1 / 3}
$$

where $n_{b}$ and $n_{p}$ are the beam and plasma electron densities, respectively. Much of the preliminary research effort consisted of the development of an electron emission algorithm for the existing particle-in-cell code, CCUBE, ${ }^{2-4}$ and of numerical simulations of elementary shaped foilless diodes. The simulations have included parameter studies of the external magnetic field and anode shaping. 5

For truncated coaxial diodes, the results indicate that for $w_{c} \leqslant u_{b}$, the diode current is limited by the impedance of the transmission line supplying power to the diode. Defining $B_{z}$ as the external magnetic field, $w_{C}=e_{z} / m_{0} c$ is the cyclotron frequency, $w_{b}=\left(4 \pi n_{b} e^{2} / m_{0}\right)^{1 / 2}$ is the beam plasma frequency, $n_{b}$ is the beam electron density, $e$ is the magnitude of the electron charge, $m_{0}$ is the electron rest mass, and $c$ is the speed of light. If the applied magnetic field strength is increased such that $w_{c}>w_{b}$, the electron beam is confined to a radius sufficiently small compared to the anode radius that the total current that can propagate down the evacuated drift tube is limited by spacecharge. However, the current density appears to be limited only the thickness of the annular beam. In fact, the space-charge limiting current actually increases as the current density increases, that is, as the thickness of the annular beam decreases.

Current densities exceeding $200 \mathrm{kA} / \mathrm{cm}^{2}$ have been obtained in the simulations at $5: \mathbb{N}$, without any special design effort or recourse to adiabatic compression of the beam. Because of the relative ease with which one can produce a high-density beam from a foilless diode, it is proposed that the major research effort be directed toward improving beam quality, as the elementary diode designs have produced beams with an angular scatter of about 100 mrad. For most applications a scattering angle of $30 \mathrm{mrad}$ or less is desirable. The source of the beam scatter appears to be caused by a lack of beam equilibrium at the emission surface, and it is felt that careful design of the 
anode and cathode shape can greatly reduce the source of beam scatter. A combined analytical and fully îme-dependent simulation effort is proposed.

Although current densities exceeding $200 \mathrm{kA} / \mathrm{cm}^{2}$ have been obtained, the investigation should also include an effort to increase the beam density because it now appears possible with careful diode design coupled with beam compression to profuce current densities exceeding $1 \mathrm{MA} / \mathrm{cm}^{2}$ in annular beams.

It is proposed to investigate the coaxial feed to the diode to maximize power flow to the diode while maintaining a high voltage at the cathode. The investigation considers the correct shaping of the outer conductor to overcome the mismatch in impedance between a self-magnetıcally insulated transmission line and an external-magnetically insulated transmission line.

A small effort on beam compression is also proposed. It was originally argued that a well-collimated high-voltage beam could be significantly adiabatically compressed if the compression was carricy out in vacuum. ${ }^{1}$ Preliminary simulations indicate that a 10 to 1 compression can be achieved with careful design of the diode region and shaping of the outer conductor in the compression region.

Continued funding of foilless diode studies by the AFOSR is proposed. The total cost of the 18-month study is $\$ 145500: \$ 95000$ requested from AFOSR and $\$ 50500$ contributed from LASL.

Results of the study will be reported in the scientific literature, if appropriate, and also in a document in a form specified by the AFOSR. The results of the study will be incorporated into an experimental investigation on the 7-MV Pulserad 1590 located at the Air Force Weapons Laboratory (AFWL) and on the 5.5 MV Maxibeam 5.5-150 at LASL.

\section{RESULTS OF PRELIMINARY STUDY}

Two series of simulations were performed using elementary diode designs to determine the effects of applied axial magnetic field strength and simple gecmetry changes. Specifically, the parameters of the simulations have included a 5 MV TEM pulse that is launched onto a nonemitting 37 ohm transmission line leading to a second magnetically insulated transmission line that transfers a voltage pulse to the diode, as depicted in Fig. 1. With this configuration, the effect of impedance mismatch on the voltage applied to the diode can be determined. All simulations were carried out in cylindrical coordinates 
using the fully time- and space-dependent particle-in-cell code CCUBE, which simulates space-charge limited emission from all regions of the cathode, including the shank.

In its simplest form the foilless diode consists of a coaxial transmission line with a truncated center conductor as the cathode of the diode. Electrons leave the cathode by tield emission. An axial magnetic field of sufficient strength prevents the electrons from striking the outer conductor. The simulations have modeled this type of diode for a center conductor (cathode) radius of $1 \mathrm{~cm}$ and an outer conductor (anode) radius of $1.853 \mathrm{~cm}$. Figure 2 shows the total current transmitted out of the diode as a function of applied magnetic field, obtained from the simulations. For small magnetic field strengths, the electrons are not confined and current is lost to the anode wall as shown in the particle plots of Fig. 3. The current increases with magnetic field strength to a maximum at intermediate fields, then decreases for large field strengths. In no case was the total current larger than the $135 \mathrm{kA}$, which would be obtained if the impedance of the diode were the same as the vacuum impedance of the transmission line, i.e., 37 ohms.

There has been some collaboration with the AFWL experimental program that has shown agreement between the foilless diode simulations and experiments of a similar nature. ${ }^{6}$ Figure 4 shows the results of one of these experiments. Note the current exhibits the same increase with magnetic field strength, reaches a maximum and is reduced at large magnetic fields. Because the parameters of the AFWL experiment are different from those of the simulation, the comparison is only qualitative. The experiment was performed with a voltage of $1.5 \mathrm{MV}$, an anode radius of $2.4 \mathrm{~cm}$ and a cathode radius of $1.32 \mathrm{~cm}$. Closer collaboration in this area is planned for the future.

As shown in Fig. 3, the electron heam expands as it moves away from the cathode. The amount of expansion depends on the magnetic field strength. The simulations indicate that the beam is annular and the inside radius depends only weakly on magnetic field strength. A calculation of the space-charge limited current as a function of outside beam radius for hollow beam propagating in a grounded conducting tube shows that the current depends strongly on beam radius. For large beam radii (small magnetic fields) the space-charge limit greatly exceeds the current observed in the simulations. As the radius of the beam is reduced (larger magnetic fields), the currents observed in the simulations are I very nearly equal to the calculated space-charge limit. Thus, the electron 


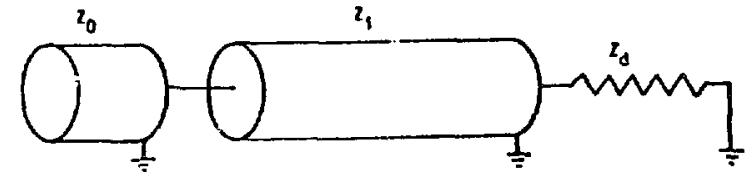

Fig. 1 .

The electrical equivalent of the circuit used in foilless diode simulations.

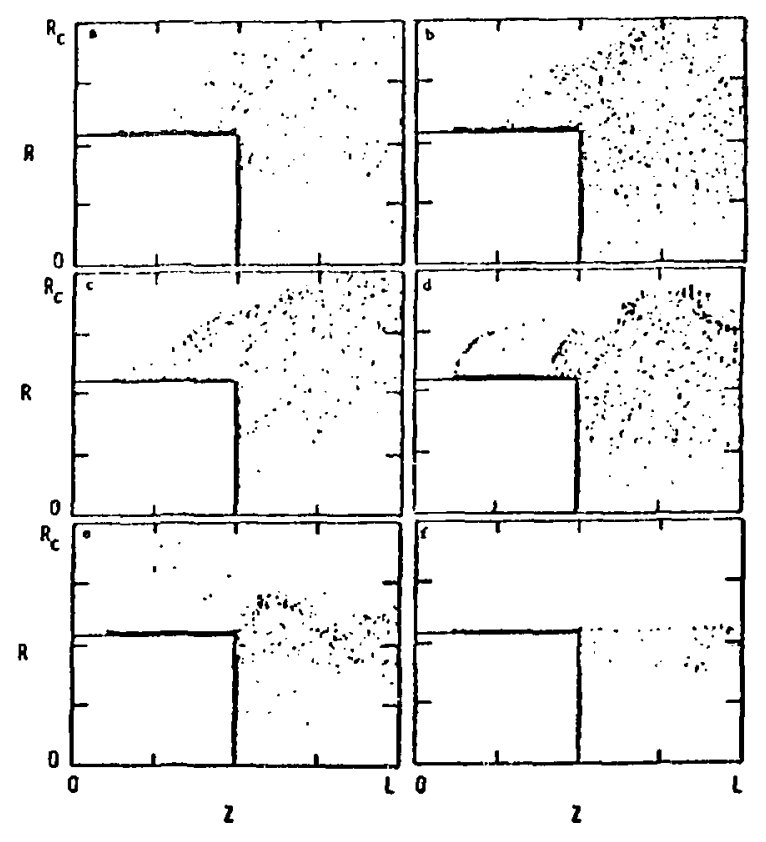

Fig. 3.

Equilibrium particle maps for various applied magnetic fields of (a) $0 \mathrm{kG}$, (b) $5.7 \mathrm{kG}$, (c) $11.4 \mathrm{kG}$, (d) $17.0 \mathrm{kG}$, (e) $28.4 \mathrm{kG}$, (f) $56.8 \mathrm{kG}$. $R_{c}=1.85 \mathrm{~cm}$ and $\mathrm{L}=5 \mathrm{~cm}$.

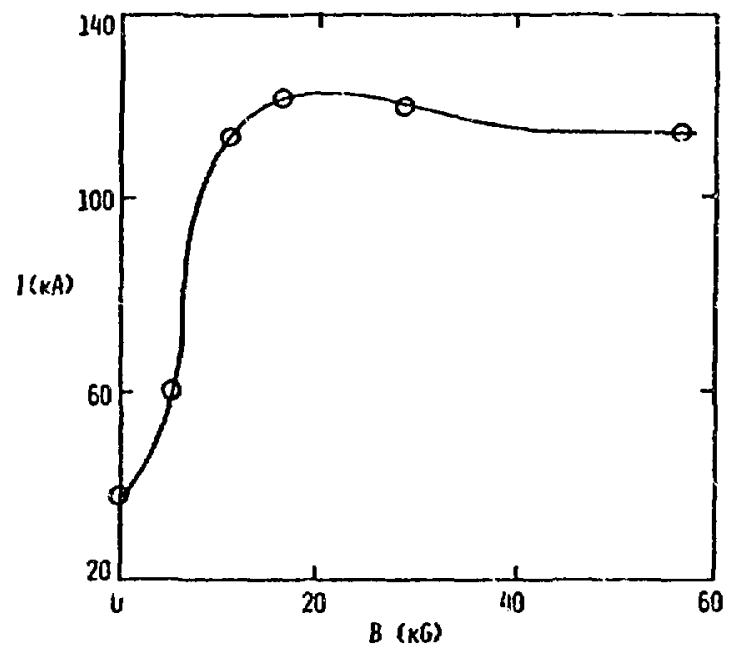

Fig. 2 .

Diode current as a function of applied magnetic field strength.

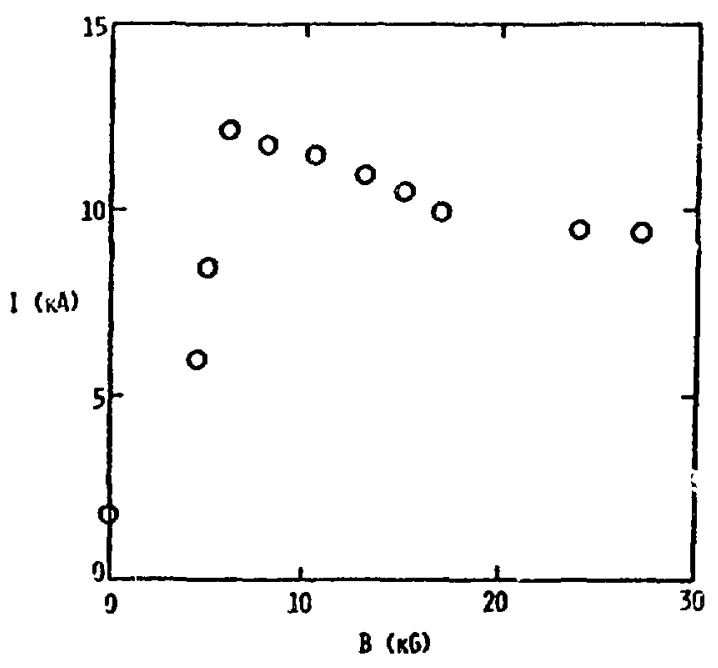

Fig. 4 .

AFWh experimental results for diode current versus applied magnetic field strength. 
current in the diode appears limited by the transmission line impedance for small magnetic field strengths and limited by space-charge for strong magnetic fields.

A second series of simulations were performed in a slightly more complicated geometry in which the anode radius changes abruptly from $1.85 \mathrm{~cm}$ to $1.13 \mathrm{~cm}$. The distance from the end of the cathode to the region of reduced anode radius was varied, and the resulting currents are shown in Fig. 5 . The solid circle corresponds to a shaped cathode. The electrode geometry and corresponding particle plots are shown in Fig. 6. These simulations indicate that the introduction of abrupt changes in geometry can lead to undesirable impedance mismatches. Although cathode and anode shaping should eventually prove to be useful, the results so far indicate that care must be taken to insure a good impedance match.

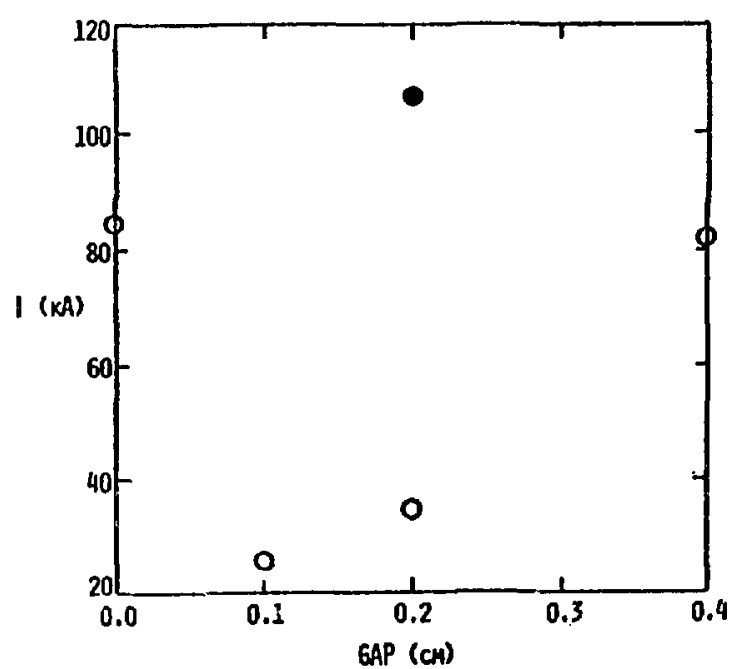

Fig. 5.

Diode current versus $A-K$ gap spacing obtained from simulations.

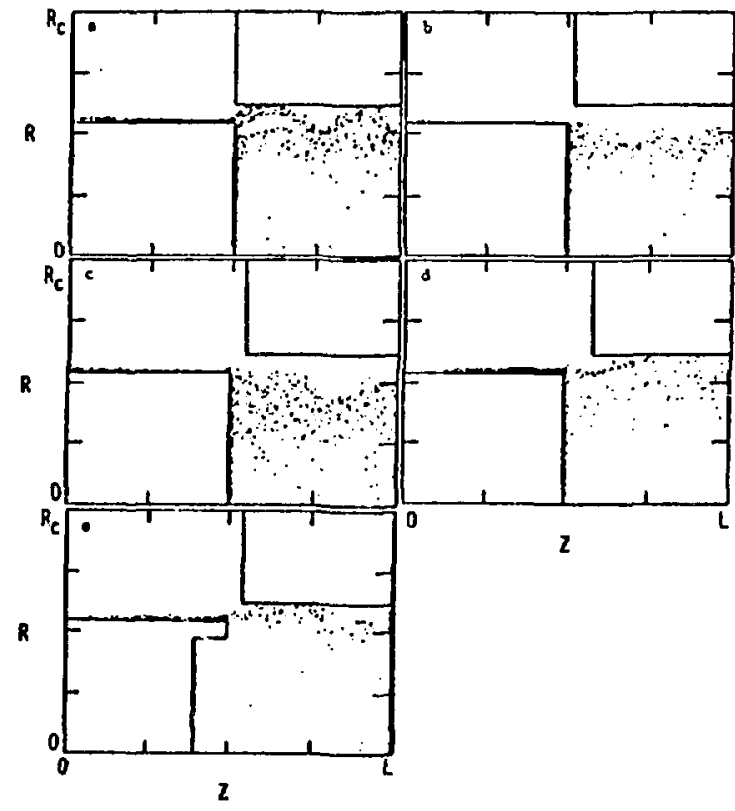

Fig. 6.

Equilibrium particle maps of various geometries. A-K gaps are (a) 0 , (b) $0.1 \mathrm{~cm}$, (c) $0.2 \mathrm{~cm}$, (d) $0.4 \mathrm{~cm}$, (e) $0.2 \mathrm{~cm}$, shaped cathode. 


\section{OBJECTIVE}

A continued investigation of foilless diode generation of a $5^{2}$ to $50 \mathrm{MeV}$ electron beam is proposed. The objective is to determine the feasibility of obtaining a beam with a local energy density of $10^{19}$ to $10^{22} \mathrm{eV} / \mathrm{cm}^{3}$, with sufficient microscopic quality to be applicable to

(1) high-density plasma heating,

(2) submillineter microwave generation, and

(3) collective ion acceleration.

\section{PROPOSED RESEARCH PROGRAM}

\section{A. Beam Equilibrium}

Emission from the cathode of the foilless diode is space-charge limited. This means the electrons are emitted with a very small initial velocity. However, a relativistic electron beam must rotate to maintain an equiliirium. Therefore, as the beam seeks an equilibrium, perturbations may be introduced that lead to thermalization of the beam. Furthermore, the presence of the cathode shorts the radial electric field at that point, a process that can launch a zero-frequency cyclotron wave on the beam. This wave is a nonpropagating wave whose wavelength, $\lambda$, is given by

$$
\lambda=2 \pi \gamma c / \omega_{c},
$$

where $w_{c}$ is the nonrelativistic cyclotron frequency, $c$ is the speed of light and $\gamma$ is the Lorentz factor. The presence of this perturbation is clearly illustrated in the particle map of a foilless diode simulation shown in Fig. $3(e)$. Thermalization from the zero-frequency cyclotron mode arises because the Lorentz factor is a function of radial position, $r$, within the beam. For example, for $a$ uniform annular beam of density, $n_{b}$, outer radius, $R_{0}$, and inner radius, $R_{i}$, the Lorentz factor is given by

$$
\gamma(r)=\gamma_{0}-2 \pi n_{b} e^{2} / m_{0}^{2}\left[\left(R_{0}^{2}-r^{2}\right) / 2+R_{i}^{2} \ln r / R_{c}+R_{o}^{2} \ln R_{c} / R_{o}\right],
$$

where $R_{c}$ is the radius of the conducting tube in which the beam propagates. Here, $\gamma_{0}=1+\mathrm{eV} / \mathrm{m}_{0} \mathrm{c}^{2}$ and $\mathrm{V}$ is the potential across the diode. Because of the radial dependence of $\gamma$, the wavelength of the zero-frequency cyclotron mode 
varies radially across the electron bean. This rosults in an effective phase mixing that leads to thermalization of the beam, the amount of thermalization depending on the amplitude of the perturbation. 7

There are several ways to minimize this type of thermalization. For instance, shaping the cathode to produce a thin beam not only increases the current density but decreases the spread in $\gamma$ across the beam. Similarly; as can be seen from Eq. (4), reducing the radius of the anode wall reduces the spread in $\gamma$. However, the simulations indicate that this must not be done abruptiy or else impedanze mismatches will occur.

To isolate the effect of varying the anode radius on beam equilibrium, a simulation was performed with a beam injected through an anode foil into a converging drift tube. The beam has an inner radius of $8 \mathrm{~mm}$ and and outer radius of $1 \mathrm{~cm}$. Because the foil shorts the radial electric field, a large amplitude zero-frequency cyclotron wave is produced. Figure 7 shows the rapid phase mixing associated with these percurbations for a beam injected into a straight drift tube, while Fig. 8 shows a reduced mixing for the beam propaga:ing into a converging drift tube. In addition, the Lorentz factor increases significantly as seen in Fig. 9, thus reducing the growth rate of the Diocotron instability.

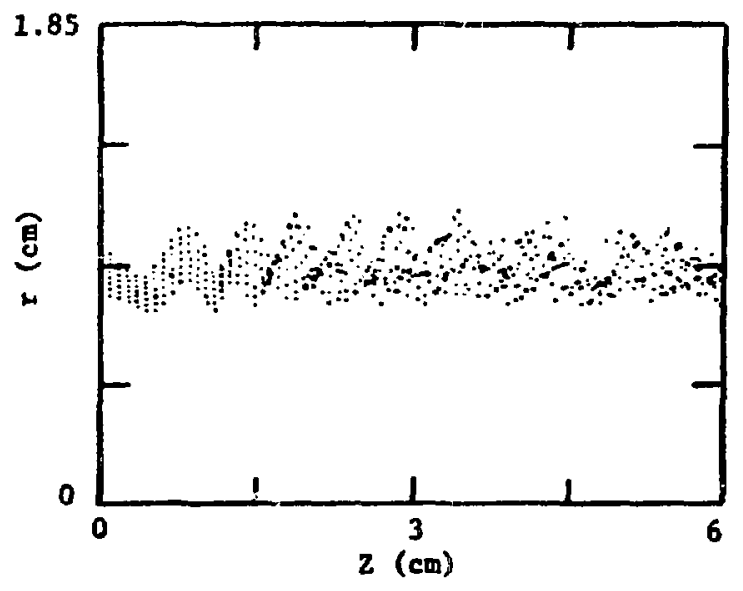

Fig. 7.

Configuration space showing the injection of a $6-\mathrm{MeV}, 75 \mathrm{kA}$ relativistic electron beam into an evacuated drift tube. Rapid phase mixing of the beam occurs in a straight drift tube.

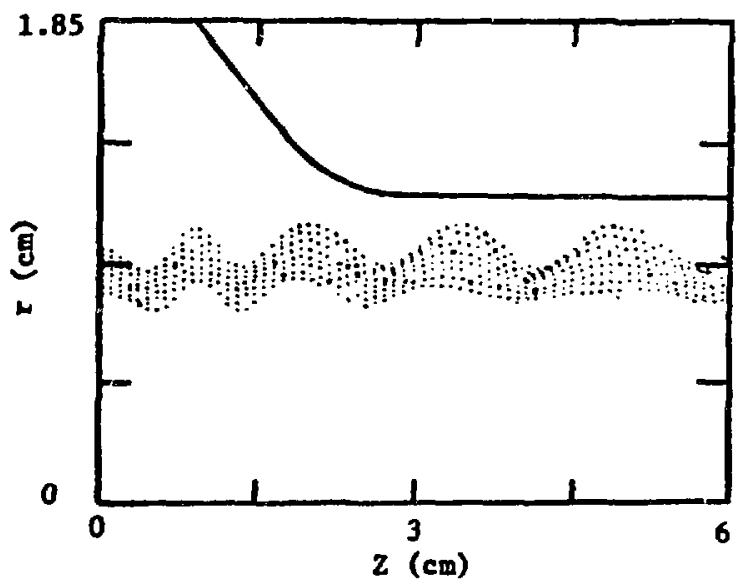

Fig. 8 .

Configuration space showing the injection of $6-\mathrm{MeV}, 75 \mathrm{kA}$ relativistic electron beam into an evacuated drift tube. The position of the converging outer conductor is indicated by the solid line. 


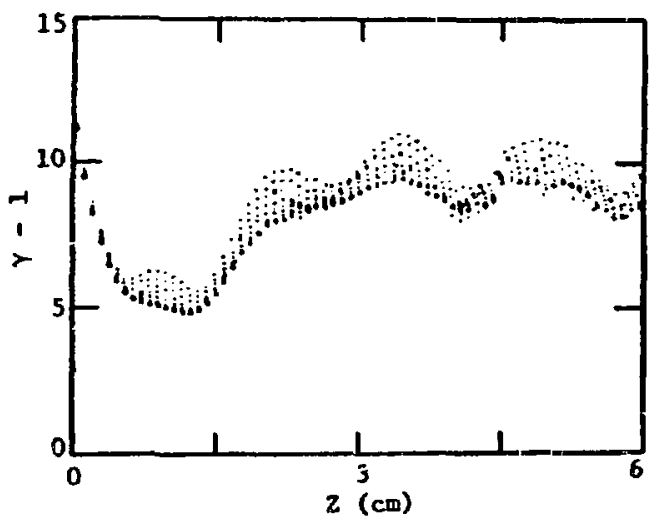

Fig. 9.

Particle kinetic energy of a $6-\mathrm{MeV}$, $75 \mathrm{kA}$ relativistic electron beam injected into an evacuated drift tube. As the beam propagates into the converging outer conductor region, the magnitude of the Lorentz factor increases.

In addition to anode shaping, shaping of the cathode can lead to a smooth launching of the beam, which reduces the amplitude of the perturbation. As pointed out by Davidson, ${ }^{8}$ there is some freedom in choosing the equilibrium of a cold relativistic electron beam. In particular, any two of the three quantities, density, axial velocity, and azimuthal velocity mas be chosen arbitrarily, and then the remaining quantity determined from Maxwell's equations and the cold fluid equations. A study of different beam equilibria may reveal that certain choices are less likely to thermalize by phase mixing. One would then try to design the diode to launch the beam into these Equilibria.

Cathode and anode shaping as well as magnetic field shaping have been used quite successfully in the design of magnetron injection guns to produce laminar electron beams. The foilless diode is the relativistic cousin of the magnetron devices. Although there have been numerous mathematical models and techniques devised, which are useful in the design of alectron beam producing devices, few of them have been extended to the case of relativistic beams. 9 The main difference between the theory of relativistic electron beams and that of the nonrelativistic case is that in the former the self magnetic field of the beam becomes important. However, there appears to be no reason why this effect cannot be properly included and the methods extended to the relativistic case. In fact, some progress has been made in this area already for the case of the Rrillouin flow model. 10,11

Much of the effort so far has been numerical. A computer simulation model has been developed, which has provided insight into the physics of diode design. The next stage of the investigation will include analytical studies to determine the shape of anode and cathode, which minimizes thermalization of the beam. The models will then be checked against the computer simulations and against experiments planned at AFWL and LASL. 
B. Coaxial Feed

To obtain a well-collimated relativistic electron beam from a highimpedance foilless ciode, it is necessary to understand the power flow through the coaxial feed. In particular, a constant impedance along the entire coaxial feed is desired. Because well-collimated electron beams require externa: miagnetic fields in the region of the diode, the region of the coaxial feed near the diode will be immersed in an axially-dependent external magnetic field, as depicted in Fig. 10. In the figure, region I represents a self-magnetically insulated lengti of coax, region II represents a transition section in which the external magnetic field is axially dependent, and region III represents an external-magnetically insulated length of coax.

Self-magnetically insulated coaxial lines have been investigated extensively. Recent work by VanDevender and McDaniel ${ }^{12}$ indicates that the impedance of the transmission line must be carefully matched to the load impedance. In particular, if the load impedance exceeds the self-magnetically insulated transmission line impedance, excess electron current is lost to the outer conductor resulting in substantial power loss. Foukey and Bergeron ${ }^{13}$ have carried out a number of time-dependent simulations of self-magnetically insulated coaxial transmission lines. For a line voltage between 1 and $10 \mathrm{MV}$, their results indicate that the self-insulated line impedance is

$$
z=(0.82 \pm 0.02)\left[\left(\gamma_{0}-1\right) /\left(\gamma_{0}+1\right)\right]^{1 / 2} z_{0}
$$

where $\gamma_{0}=1+e V / m_{0} c^{2}, V$ is the applied line voltage, and $z_{0}$ is the geometrical impedance of the coaxial line.

We have performed four self-magnetically insulated coaxial transmission line simulatinns, in which $a=1 \mathrm{~cm}, \mathrm{R}=1.853 \mathrm{~cm}, L=60.0 \mathrm{~cm}$, and $Z_{0}=37 \Omega$. For $1.5,5,6$, and $20 \mathrm{MV}$ TEM pulses our simulations agree with Eq. (5), see Tabie I. Thus, the impedance of self-magnetically insulated coaxial transmission lines is always less than the geometrical impedance of the line. In carrying out foilless diode simulations, it appears that the impedance of an external-magnetically insulated roaxial transmission line approaches the geometrical impedance as the external magnetic field strength is increased. Thus, the selfmagnetically insulated line shown in Fig. 10 is overmatched and will result in a loss of electrons to the outer wall. Electrically, the configuration shown 
TABLE I

COAXIAL TRANSMISSION LINE IMPEDANCE

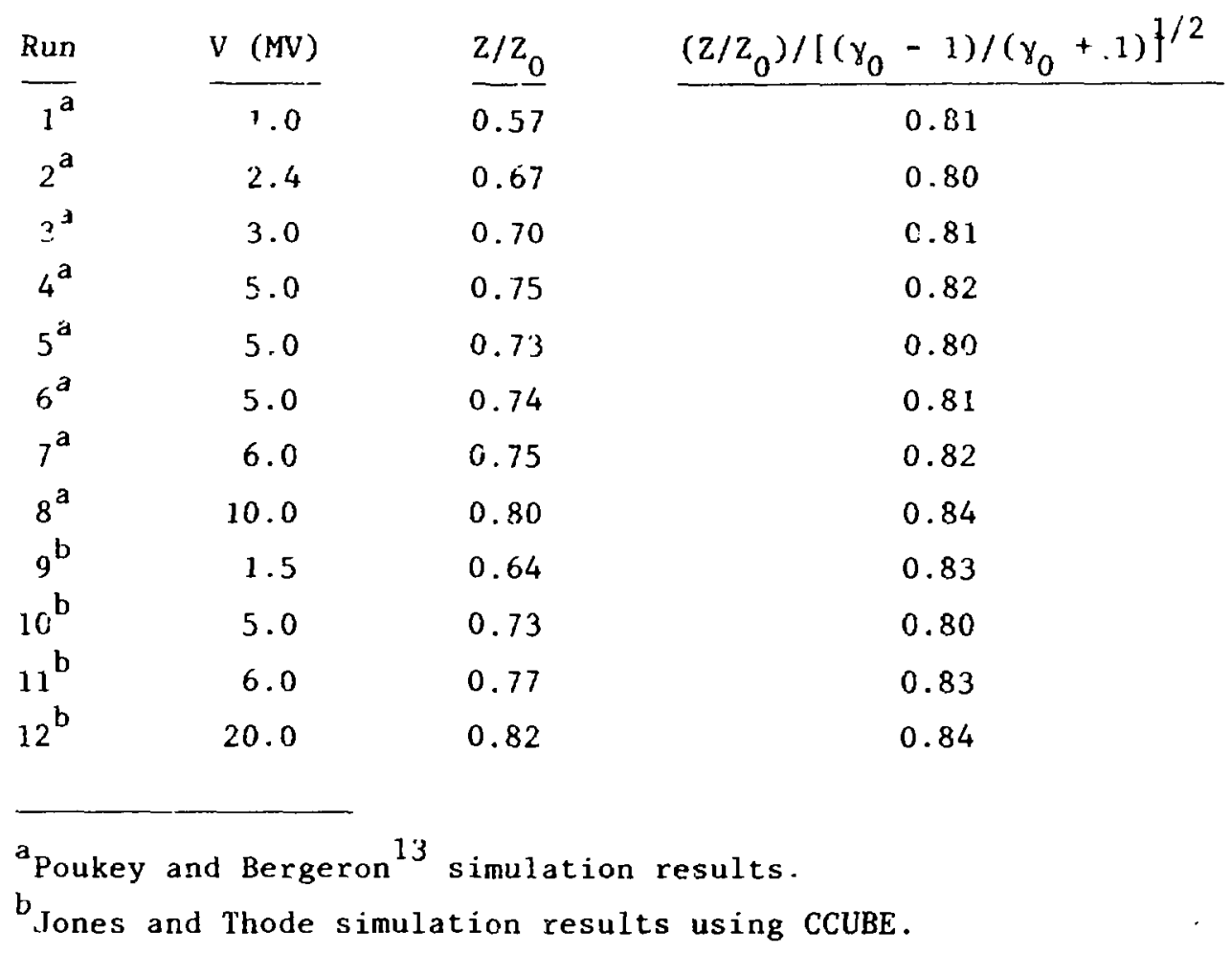

in Fig. 10 appears as illustrated in Fig. 11. The impedance of the short transmission lines between the self $\cdots$ insulated line and the external-insulated line depend upon the magnitude of the local external magnetic field $\mathrm{E}_{Z}$ and the geometrical ratio $\mathrm{R} / \mathrm{a}$. If the impedance is to be matched, $\mathrm{R} / \mathrm{a}$ must be reduced as $\mathrm{B}_{\mathrm{Z}}$ increases, as shown in Fig. 12. To achieve the proper configuration, the impedance as a function of $B_{Z}$ must be determined. If the change in the external magnetic field strength over an electron larmor orbit is small, then it would be possible to obtain $2\left(B_{0}\right)$ by carrying out a large number of small simulations. However, as an initial approach it appears that an approximate analytical solution of $Z\left(B_{0}\right)$ could be obtained. Simulations incorporating the required wall radius as a function of the axial position could then be carried out for the matched line. Ultimately, the impedance should drop slightly through the transition region, to insure that the self-magnetically insulated driving line remains undermatched for as much of the pulse as possible. 


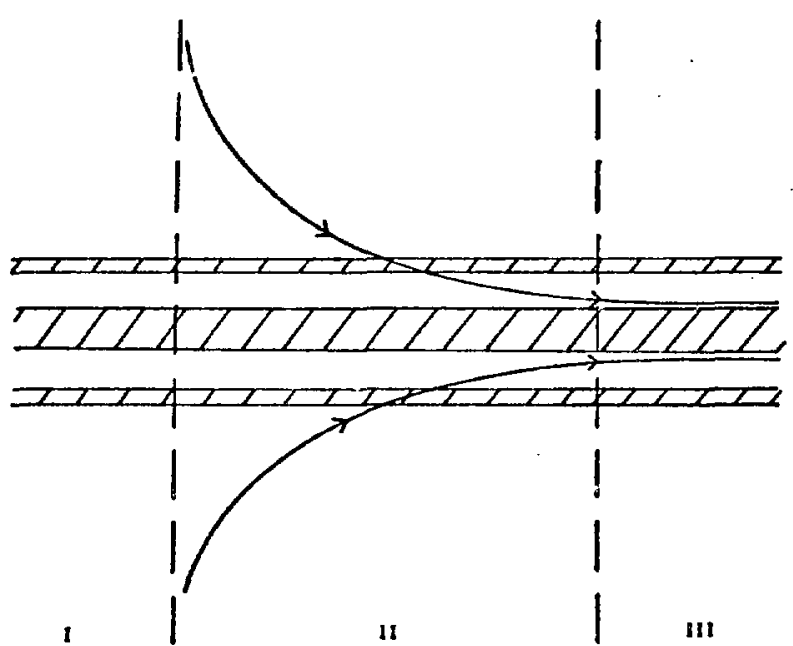

Fig. 10 .

Illustration of the three regions of a coaxial feed for a foilless diode.

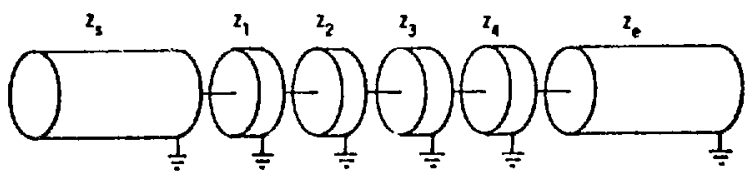

Eig. 11 .

Electrical equivalent of coaxial feed with an external magnetic present at the diode. The impedance of $Z_{1}$ through $Z_{4}$ depends upon the local value of the external rield $\mathrm{B}_{Z}$ and the geometrical ratio of $\mathrm{R} / \mathrm{a}$.

\section{Adiabatic Compression}

It was originally argued that significant adiabatic compression could be achieved for a well-collimated, high-voltage electron beam if the compression was carried sut in vacuum. ${ }^{1}$ The major question, however, concerned the tradeoff in beam quality between current density at the diode and the adiabatic compression ratio of a fixed-beam energy density at the exit of the compression region. Based upon the preliminary simulation results, a current density exceeding $200 \mathrm{kA} / \mathrm{cm}^{2}$ can be obtained at $5 \mathrm{MV}$. However, beam collimation is not sufficient for the desired application and adiabatic compression will only further scatter the beam.

If through shaping of the cathode and the outer conductor wall it becomes possible to reduce the angular scatter to $10 \mathrm{mrad}$, compression ratios of 10 to 1 can be carried out. For example, in Fig. 13 a $6 \mathrm{MeV}$ annular electron beam of inner radius $8 \mathrm{~mm}$ and outer radius $1 \mathrm{~cm}$ is injected into a 10 to 1 compression field. The beam had an initial scatter of $10 \mathrm{mrad}$ increasing to $35 \mathrm{mrad}$ after being compressed. The only problem appears to be the correct shape of 


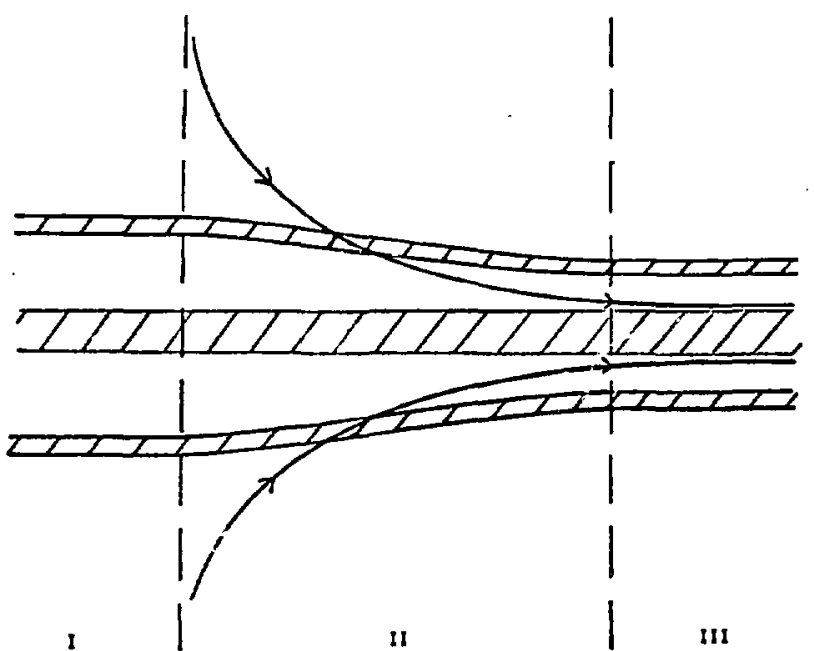

Fig. 12.

Illustration of an impedance matched coaxial feed for a foilless diode.

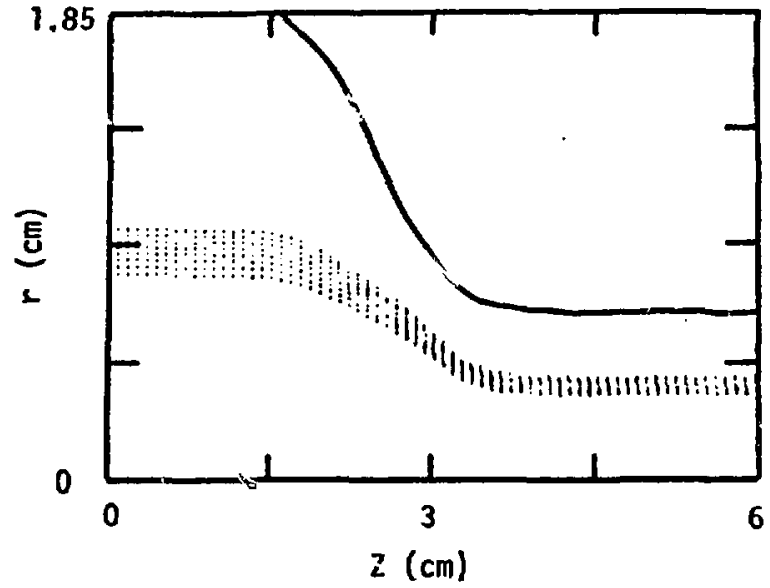

Fig. 13 .

Configuration space showing the adiabatic conpression of a $6-\mathrm{MeV}, 75 \mathrm{kA}$ relativistic electron beam in an evacuated drift tube. The position of the converging outer conductor is indicated by the solid line.

the other conductor in the compression region. Ideally, the separation between the outer beam radius and the wall should decrease through the compression region. This is not the case in the present example, in which the outer conductor shape was determined by magnetic flux conservation. Based upon this simulation and present magnetic field technology, ${ }^{14}$ it is proposed to only investigate the size of the magnetic field gradient required to maintain laminar flow.

D. Code Development

The particle-in-cell simulation code, CCUBE, now provides a fully relativistic time- and space-dependent treatment of space-charge limited diode emission. At present only simple cathode and anode shapes can be treated. Some of the future studies will require the code to be modified to allow treatment of more general electrode designs. Improvements will be made on the treatment of applied external magnetic fields. Diagnostics will probably need to be added and modified for proper evaluation of diode designs. 
V. WORK STATEMENT

The investigation shall include a study of the effects of shaping the cathode, the anode, and the applied magnetic field. The main emphasis of this work will be on obtaining a general understanding of diode design, which of'linizes the laminar flow of the beam. Analytical creatments of these effects will be made to as great an extent as possible. The results of the analytical treatments $w 11$ be tested against simulations obtained from CCUBE and against available axperimental results. Madifications and improvements in the simulation code will be made as necessary to evaluate the diode designs.

Fifects of emission along the coaxjal line leading to the cathode will be studied. In particular, the effects of the varying external magnetic along this line and how it impacts diode performance will be investigated.

Adiabatic compression of the beam will be studied to determine the size of the gralient in field strength ruquired to maintain laminar flow.

VI. TERM

The work will be initiated on April 1, 1979, or upon receipt of authorization from the Department of Energy to proceed, whichever is later. The duration of the proposed study is 18 months. If authorization is not received by August 1, 1979, it may become necessary to modify this proposal.

VII . REPOR'TING

Results of the study will be reported as required by the AFOSR. If appropriate, the results of the study will also be reported in the scientific li terature.

VI I I . PERSONNEL

L. E. Thode of Group T-15 (LASL) wiil be the manager of the proposed study. In collaboration with Thode, M. E. Jones of Group T-15 (IASL) will have the general responsibility for the performance and reporting of the proposed work, and for liaison with the Air Force representatives as required.

In addition to Jones and hode, B. Godfrey, J. Cary, R. Faehl, T. Kwan, M. Mostrom, B. Newberger, and R. Shanahan of Group T-15 (LASL), and C. Clark of AFWL will act as technical consultants for the proposed study. 


\section{REFERENCES}

1. Lester E. Thode, "A Proposal for Study cf Vacuun Adiabalic Compression of a Relativistic Electron Beam Generated by a Foilless Diode," Los Alamos Scientific Laboratory report LA-7169-P (February 1978).

2. B. B. Godfrey and L. E. Thode, "Galerkin Difference Schemes for Plasma Simulation Codes," Proc. Seventh Conf. Num. Sim. Plas. (Wew York, 1975) p. 87-91.

3. B. B. Codfrey, "Application of Galerkin's Method to Particle-In-Cell Plasma Simulation," Proc. Eighth Conf. Nun. Sim. Plas. (Monterrey, 1973), paper PE -3

4. B. B. Godfrey, "A Galerkin Algorithm for Multi-Dimensional Plasme Simulaijon Codes," Bull. An. Phys. Soc. 23, 841 (1978).

5. Michael E. Jones and Lester E. Thode, "Numerical Simulations of Coaxial Foilless Diodes," Bull. Am. Phys. Soc. 23, 839 (1978).

6. D. C. Straw and M. C. Clark, "Foilless Diode Behavior in a Strong External Magnetic Field," Bull. Am. Phys. Soc. 23, 839 (1978).

7. L. E. Thode, B. B. Godfrey, and W. R. Shanahan, "Vacuum Propagation of Solid Relativistic Electron Beams: Correlations Among Experiment, Simulation, and Theory," to be published in Phys. Fluids (April 1979).

8. R. (.. Davidson, Theory of Nonneutral Plasmas (Benjamine, Massachusetts, 1974).

9. P. T. Kirstein, G. S. Kino, and W. E. Waters, Space Charge Flow (McGrawHill, New York, 1967).

10. J. M. Creedon, "Relativistic Brillouin Flow in the High v/Y Diode," J. Appl. Phys. 46, 2946 (1975).

11. J. M. Creedon, "Magnetic Cutoff in High-Current Diodes," J. Appl. Phys. 48, $1070(1977)$.

12. J. P. VanDevender and D. H. McDaniel, "Advanced Power Flow Technologies for High Current ICF Accelerators," to be published.

13. J. W. Poukey and K. D. Bergeron, "Space-Charge Effects in Long Coaxial Vacuum Transmission Lines," Appl. Phys. Lett. 32, 8 (1978).

14. H. P. Furtl, M. A. Levine, and R. W. Wanick, "Production and use of High Transient Magneti- Fields. II," Rev. Sci. Inst. 28, 949 (1957). 